\title{
The role of systematic uncertainties on our understanding of cosmic-ray diffusion: An analysis of AMS-02 data from Lithium to Oxygen
}

\author{
Michael Korsmeier ${ }^{a, *}$ and Alessandro Cuoco ${ }^{b, c}$ \\ ${ }^{a}$ The Oskar Klein Centre, Department of Physics, Stockholm University, \\ AlbaNova, SE-10691 Stockholm, Sweden \\ ${ }^{b}$ Dipartimento di Fisica, Università di Torino, \\ Via P. Giuria 1, 10125 Torino, Italy \\ ${ }^{c}$ Istituto Nazionale di Fisica Nucleare, Sezione di Torino, \\ Via P. Giuria 1, 10125 Torino, Italy \\ E-mail: michael.korsmeier@fysik.su.se, alessandro.cuoco@unito.it
}

In the last decade, the space-borne experiment AMS-02 has determined cosmic-ray spectra with unprecedented precision, potentially providing new insights into cosmic-ray propagation in our Galaxy. However, the analysis of this increasingly precise cosmic-ray data requires more careful modeling of systematic uncertainties. We analyze the recent AMS-02 comic-ray measurements of the secondaries Lithium, Beryllium, and Boron, and the primaries Carbon, Nitrogen and Oxygen. The data is used to study five different propagation frameworks, which for example differ by including or neglecting reacceleration or breaks in the diffusion coefficient. In this work, we particularly focus on systematic uncertainties related to the fragmentation cross section to produce secondary CRs. Furthermore, the impact of correlated systematic uncertainties in the AMS-02 data are studied. The cosmic-ray data from Li to $\mathrm{O}$ is well fitted in all the considered propagation frameworks. However, we find that the uncertainties on the nuclear production cross sections play a crucial role. Because of these uncertainties we cannot distinguish between different propagation frameworks. In this sense, cross section uncertainties currently prevent a deeper understanding of the properties of CR propagation. Nonetheless, we find some common and robust constraints. Above a few GV, the slope of the diffusion coefficient is determined to be in the range of $\delta \simeq 0.4-0.5$. Moreover, we find a lower bound on the half-height of the diffusion halo at $z_{\mathrm{h}} \gtrsim 3 \mathrm{kpc}$.

$37^{\text {th }}$ International Cosmic Ray Conference (ICRC 2021)

July 12th - 23rd, 2021

Online - Berlin, Germany

\footnotetext{
${ }^{*}$ Presenter
} 


\section{Introduction}

Historically, $\mathrm{B}$ and the $\mathrm{B} / \mathrm{C}$ ratio have been the most investigated nuclei to constrain cosmic-ray (CR) propagation. Nonetheless, the precise CR data in a large energy range between $1 \mathrm{GeV}$ and a few TeV provided by AMS-02 [1] have triggered a great number of new studies [2-9]. We perform global fits of CR propagation models to the published AMS-02 data of CR secondaries $\mathrm{Li}, \mathrm{Be}$, and $\mathrm{B}$ [10], the primaries $\mathrm{C}$ and $\mathrm{O}$ [11], and the mixed secondary and primary $\mathrm{N}$ [12]. Using the precise data we investigate five different setups of CR propagation, which differ with respect to the inclusion of a break in the diffusion coefficient and/or in the injection spectrum at a few GV, and the presence of diffusive reacceleration. The most important novelty of this work is a rigorous inclusion of systematic uncertainties related to fragmentation cross-section to produce secondary CRs. These systematic uncertainties are at the oder of 20 to $30 \%$ which is a factor of a few larger compared to the uncertainties of the AMS-02 flux measurements.

\section{Cosmic-ray propagation}

In the following, we briefly summarize our general prescription of CR propagation and the specific assumptions of the five setups. For a more detailed description we refer to [13]. The propagation of Galactic CRs is described in terms of a chain of coupled diffusion equations of the form [14]:

$$
\begin{aligned}
\frac{\partial \psi_{i}(\boldsymbol{x}, p, t)}{\partial t}=q_{i}(\boldsymbol{x}, p) & +\boldsymbol{\nabla} \cdot\left(D_{x x} \boldsymbol{\nabla} \psi_{i}-\boldsymbol{V} \psi_{i}\right) \\
& +\frac{\partial}{\partial p} p^{2} D_{p p} \frac{\partial}{\partial p} \frac{1}{p^{2}} \psi_{i}-\frac{\partial}{\partial p}\left(\frac{\mathrm{d} p}{\mathrm{~d} t} \psi_{i}-\frac{p}{3}(\boldsymbol{\nabla} \cdot \boldsymbol{V}) \psi_{i}\right)-\frac{1}{\tau_{f, i}} \psi_{i}-\frac{1}{\tau_{r, i}} \psi_{i} .
\end{aligned}
$$

Here $\psi_{i}$ is the number of CR species $i$ per volume and total momentum. In general, we distinguish between primary and secondary CR nuclei. Primary nuclei are produced at astrophysical sources like supernova remnants (SNRs) or pulsars. Their source term is modeled as a smoothly-broken power law in rigidity. The spectral indices above and below the break at the rigidity $R=R_{\text {inj, } 0}$ are labeled $\gamma_{1}$ and $\gamma_{2}$. The parameters $s$ regulated the amount of smoothing. Spatially it follows the distribution of SNRs [15]. The overall normalization of the primary source terms is called the isotopic abundance (in plots abbreviated by Iso. Ab.), which is fixed relative to an arbitrary proton abundance of $10^{6}$. On the other hand, secondary nuclei are mostly produced by the fragmentation of primary CRs on the interstellar medium (ISM) which leads to a different source term:

$$
q_{i, \mathrm{sec}}\left(R_{i}\right)=\left.\sum_{k>i} \sum_{j=\{\mathrm{p}, \mathrm{He}\}} 4 \pi n_{\mathrm{ISM}, j} \phi_{k}\left(R_{k}\right) \sigma_{k+j \rightarrow i}\left(T_{k}\right)\right|_{\frac{T_{k}}{A_{k}}=\frac{T_{i}}{A_{i}}} .
$$

Here the fragmentation cross section of CR species $k$ to $i$ on the ISM component $j$ plays an important role. It is generally assumed that the kinetic energy per nucleon is preserved during fragmentation $\left(T_{k} / A_{k}=T_{i} / A_{i}\right)$. Above a few tens of GV, CR propagation is dominated by diffusion. The diffusion coefficient $D_{x x}$ generally increases with energy. We use a double-broken power law in rigidity with break positions at $R=R_{D, 0}$ and $R_{D, 1}$ to model the diffusion coefficient. The power-law indices below, in between, and above the breaks are called $\delta_{l}, \delta$, and $\delta_{h}$, and the two parameters $s_{D, 0}$ and 
$s_{D, 1}$ allow for a smoothing around the breaks. Furthermore, we allow for constant convective winds that drive the CRs away from the Galactic plane. In some cases, diffusive reacceleration is allowed in our models. Then, its amount is parametrized in terms of the velocity $v_{\mathrm{A}}$ of Alfvèn magnetic waves $[16,17]$. The last two terms of Eq. (1) describe the fragmentation and decay processes with the respective timescales $\tau_{f, i}$ and $\tau_{r, i}$. We use the GALPROP $\operatorname{code}^{1}[18,19]$ with some custom modifications for our analysis to solve the diffusion equations of CRs numerically We assume a steady state solution and cylindrical symmetry of our Galaxy with the Sun located at a distance of $8.5 \mathrm{kpc}$ from the center. Finally, solar modulation is treated in the force-field approximation [20], which relates the interstellar flux and the flux at the top of the atmosphere through a single parameter, commonly known as the force-field potential $\varphi_{\mathrm{SM}, i}$. Its strength varies during the 22-year solar cycle.

\subsection{Secondary production cross section}

Secondary nuclei are mostly produced by fragmentation of primary nuclei on the ISM. Here we consider the secondary nuclei $\mathrm{Li}, \mathrm{Be}$, and $\mathrm{B}$. While Carbon and $\mathrm{O}$ make up the largest fraction of their production, we consider nuclei up to $\mathrm{Si}$ in the GALPROP simulation. For each species there are several reactions and isotopes that contribute. For example, B has two stable isotopes, ${ }_{5}^{10} \mathrm{~B}$ and ${ }_{5}^{11} \mathrm{~B}$ with a relative contribution of $1 / 3$ and $2 / 3$, which originate mostly from the fragmentation of the stable $\mathrm{C}$ (about $90 \%{ }_{6}^{12} \mathrm{C}$ and $10 \%{ }_{6}^{12} \mathrm{C}$ ) and $\mathrm{O}$ (mostly ${ }_{8}^{16} \mathrm{O}$ ) nuclei. The large number of fragmentation cross sections with scarce and often not very precise measurements constitute a major uncertainty in the prediction of the secondary CR fluxes [21]. We found that it is essential to take these uncertainties into account to avoid biased conclusions. From Ref. [21] we identified the 16 most important reactions and introduced nuisance parameters to capture the uncertainty. The nuisance parameters allow to change the normalization of the cross section, labeled $A_{\text {reaction }}$, and its slope below a kinetic energy of $T_{\text {ref }}=5 \mathrm{GeV} /$ nuc, labeled $\delta_{\text {reaction: }}$ :

$$
\sigma_{k+j \rightarrow i}\left(T_{k} / A\right)=\sigma_{k+j \rightarrow i}^{\text {default }}\left(T_{k} / A\right) \cdot A_{k+j \rightarrow i} \cdot\left\{\begin{array}{ll}
\left(T_{k} / T_{\text {ref }}\right)^{\delta_{k+j \rightarrow i}} & T_{k} / A<T_{\text {ref }} / A \\
1 & \text { otherwise }
\end{array} .\right.
$$

Here $\sigma_{k+j \rightarrow i}^{\text {default }}$ is the default cross section GALPROP for the fragmentation of a CR species $k$ on the ISM component $j$ to produce the CR species $i$. This would correspond to a huge number of 32 nuisance parameters and it is technically not feasible to add such a large number of parameters to the fit. Instead, we group the nuisance parameters according to the five fragmentation product ( $\mathrm{Li}$, $\mathrm{Be}, \mathrm{B}, \mathrm{C}$, and N) and vary only one single parameter to capture the overall effect. This leads to a total of 10 nuisance parameters. More details are given in [13] (cf. Tab. II).

\subsection{Five specific setups of $\mathrm{CR}$ propagation}

The general setup of CR propagation is described at the beginning of Sec. 2. In the following, we will consider five specific scenarios, which are special cases of the general one. The simplest setup is called BASE. Here we use a simple (unbroken) power law for the injection spectrum, i.e. $\gamma_{1}=\gamma_{2}$. Thus, the break parameters $R_{\mathrm{inj}, 0}$ and $s$ become uninfluential. Furthermore, reacceleration

\footnotetext{
${ }^{1}$ More specifically, we use GalPROP version 56.0.2870 and Galtoollibs 855, both available here.
} 
is not included. This setup contains a total of 23 free parameters, 13 parameters describing CR propagation and ten nuisance parameters related to the uncertainties of the fragmentation cross sections. We then consider the following extensions of the BASE setup: The BASE $+v_{A}$ setup includes reacceleration, the BASE+inj setup includes a break in the injection spectrum, and the BASE $+\mathrm{inj}+v_{A}$ setup includes both. This leads to one, three, and four additional fit parameters, respectively. Finally, the setup dubbed BASE + inj $+v_{A}-$ diff.brk includes both reacceleration and a break in the injection spectrum, but it does not have the break in the diffusion coefficient. It has one parameter more than the BASE setup. In all cases, we fix the radial extension of the diffusion halo to $20 \mathrm{kpc}$ and, unless stated differently, its half-height to a value of $4 \mathrm{kpc}$. The latter is justified by the well-known degeneracy between the half-height of the diffusion halo and the normalization of the diffusion coefficient. We will explore constraints on the halo height at the end of the results section.

\subsection{Fit method}

We use the MultiNest algorithm [22] to sample the CR log-likelihood ${ }^{2}$ defined as the sum of the $\chi^{2} \mathrm{~s}$ of the individual species:

$$
-2 \log \left(\mathcal{L}_{\mathrm{CR}}\right)=\chi_{\mathrm{CR}}^{2}=\sum_{s} \sum_{i, j}\left(\phi_{s, i}-\phi_{s}^{(\mathrm{m})}\left(R_{i}\right)\right)\left(\left(\mathcal{V}^{(s)}\right)^{-1}\right)_{i j}\left(\phi_{s, j}-\phi_{s}^{(\mathrm{m})}\left(R_{j}\right)\right) .
$$

Here $\phi_{s, i}$ is the flux of the CR species $s$ measured by AMS- 02 at the rigidity $R_{i}$ and $\phi_{s}^{(\mathrm{m})}$ is the corresponding model prediction taken from GALPROP. $\mathcal{V}^{(s)}$ is the covariance matrix containing the uncertainty of the flux measurement. Unless stated explicitly, we assume uncorrelated uncertainties, i.e., $\mathcal{V}_{i j}^{(s)}=\delta_{i j}\left(\sigma_{s, i}\right)^{2}$, with $\sigma_{s, i}$ being the error reported by AMS-02, more precisely, with statistical and the systematic error added in quadrature. The solar modulation potential is a free parameter in the fit, but we add a Gaussian prior with a mean of $600 \mathrm{MV}$ and a width of $30 \mathrm{MV}$. As an additional cross check, we will explore the impact of correlated systematics in the experimental uncertainties (labeled corr in plots). The correlations are not provided by the AMS-02 collaboration, so we follow the approach of Ref. [23] to model the correlation matrix. For more details we refer to the Refs. [13, 23, 24]. All results are shown and interpreted in a frequentist framework. We derive our results both for a fit to a reduced data set of $\mathrm{B}, \mathrm{C}, \mathrm{N}$, and $\mathrm{O}$, called $\mathrm{BCNO}$ and for the full data set, labeled LiBeBCNO.

\section{Results}

We obtain a good fit of the $\mathrm{Li}$ to $\mathrm{O}$ data for each of the five propagation setups, which is a remarkable result given the extremely precise AMS-02 data. The spectra of the best-fit point are compared to the measured AMS-02 data in Fig. 1. The good quality of the fit is apparent by looking at the residuals. Note that we do not show the residuals for the cases of correlated uncertainties (dashed lines). The correlations in the data points can allow for small re-normalizations and/or tilts. Therefore, looking at the residuals might be misleading and one should directly look at the

\footnotetext{
${ }^{2} \mathrm{~A}$ few parameters do not depend on the GALPROP run. To save computing time we profile over these parameters on-the-fly. More details are given in [13].
} 

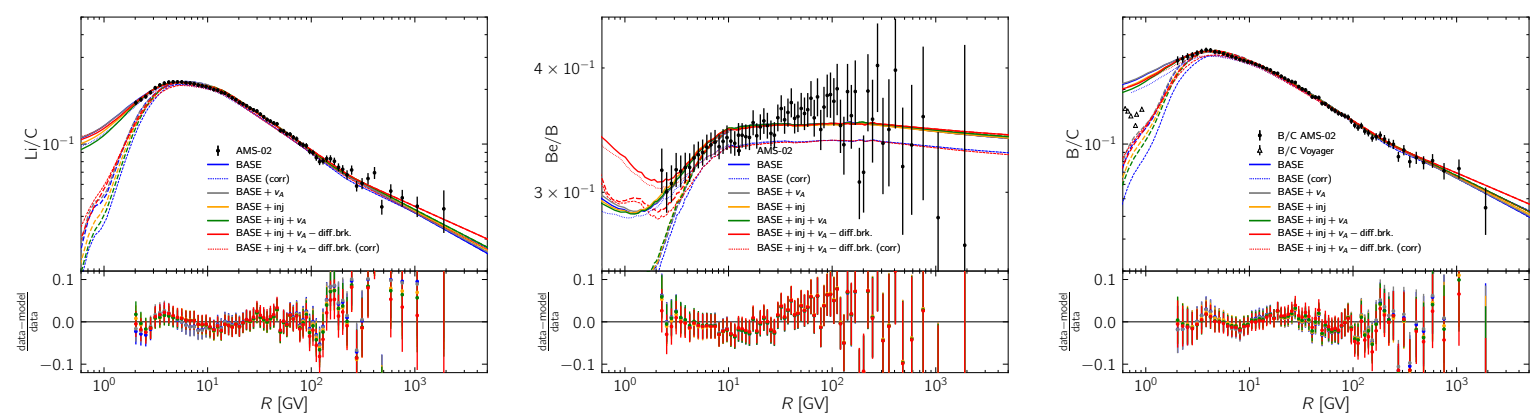

Figure 1: Comparison of a selection of AMS-02 measurements with the best-fit spectra for the LiBeBCNO data set. We show the best-fit model for each of the five propagation frameworks (solid lines) and the interstellar spectra which can be compared to the Voyager data at low energies (dashed lines). Dotted lines are the best-fit models in the scenario with correlated systematic uncertainties in the AMS-02 data. The figure is taken from Ref. [13].
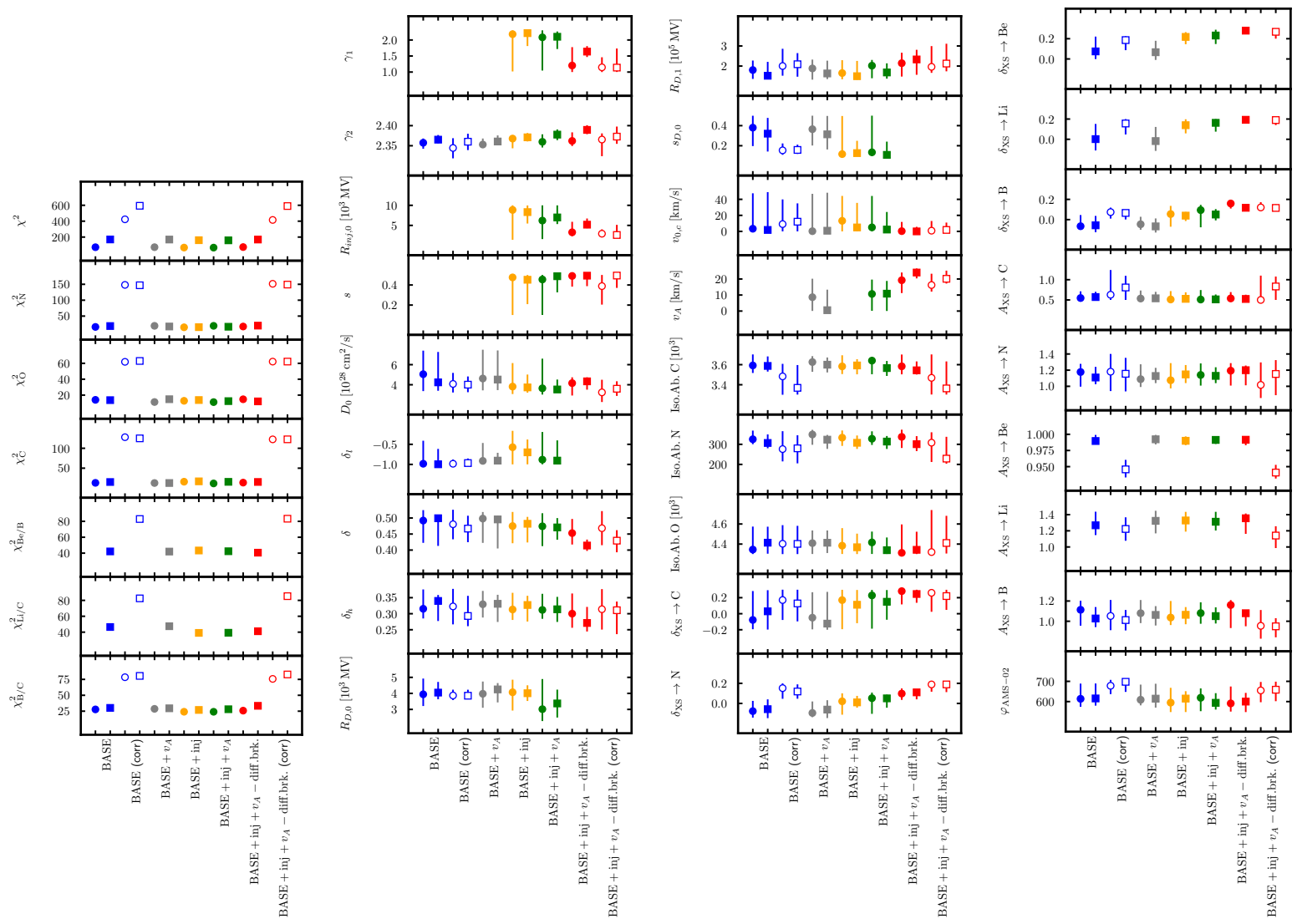

Figure 2: Best-fit values and $2 \sigma$ uncertainties for all CR propagation, cross-section nuisance, and solar modulation parameter. The values of different $\mathrm{CR}$ propagation setups are distinguished by color. Circles (squares) refer to the $\mathrm{BCNO}$ ( $\mathrm{LiBeBCNO}$ ) data set. Filled data points refer to the standard case of uncorrelated AMS-02 systematic uncertainties, while open data points are used for the cases where correlated systematics are considered. The figure is taken from Ref. [13].

$\chi^{2}$-values, reported in the first column of the comparison plot of Fig. 2. The remaining 3 columns of Fig. 2 show the best-fit parameter values for all the fits using different data sets and CR propagation setups. In the following we focus on the three main results and refer to [13] for a more detailed 


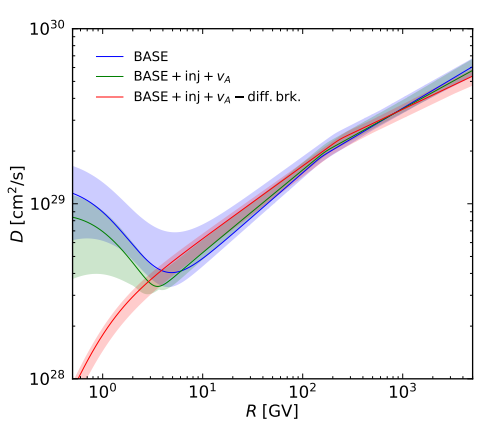

Figure 3: Diffusion coefficient as function of rigidity for three different fit configurations. The halfheight of the diffusion coefficient is fixed to $z_{\mathrm{h}}=4 \mathrm{kpc}$. The shaded band shows the $2 \sigma$ uncertainty. The figure is taken from Ref. [13].
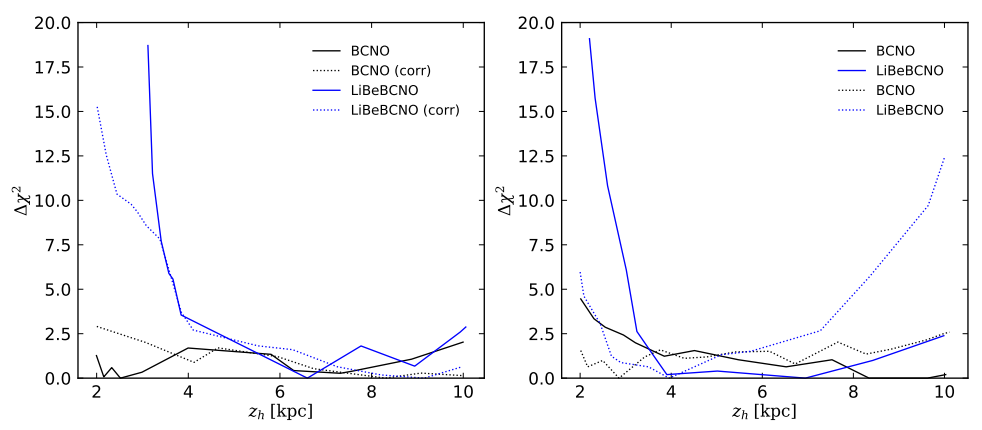

Figure 4: $\chi^{2}$ profile as function of halo half-height $z_{\mathrm{h}}$. The blue lines correspond to the full data set from $\mathrm{Li}$ to $\mathrm{O}$ while the black lines correspond to the reduced data set using only $\mathrm{B}, \mathrm{C}, \mathrm{N}$, and $\mathrm{O}$. The left panel refers to the BASE framework, while the right one refers to the BASE+inj $+v_{A}-$ diff.brk framework. Dashed lines are used when the fit assumes correlations in the AMS-02 data. The figure is taken from Ref. [13].

discussion.

All five CR propagation setups provide a very good fit to the data and result in a similar minimal $\chi^{2}$ value. The most general setup BASE+inj+ $v_{A}$ has a best-fit $\chi^{2}$ value of 159 and has 17 free propagation parameters, while BASE and BASE + inj $+v_{A}$-diff.brk with 13 and 14 propagation parameters have best-fit $\chi^{2}$ values of 170 and 169, respectively. The other $\chi^{2}$ values are in between. We note that the models BASE and BASE $+\mathrm{inj}+v_{A}$-diff.brk are not nested models, however, all models are nested in BASE $+i n j+v_{A}$. In this sense, we concluded that no model is statistically significantly preferred. A similar conclusion holds for the BCNO data set. The inclusion of cross section uncertainties are crucial to obtain this result. For the BASE setup the cross section nuisance nuisance parameters converge to their default values $\left(A_{\mathrm{XS}} \rightarrow 1\right.$ and $\left.\delta_{\mathrm{XS}} \rightarrow 0\right)$, while the $\mathrm{BASE}+\mathrm{inj}+v_{A}-$ diff.brk systematically prefer $\delta_{\mathrm{XS}}$ values incompatible with 0 and around 0.2 . These are well within the current measurement uncertainty of the cross section. This discussion shows that with a better knowledge of cross section we would be able to distinguish between the setups.

Figure 3 shows that the diffusion coefficient is very well constrained above $10 \mathrm{GV}$. If a break in the diffusion coefficient is allowed, $D_{x x}$ increases below a few GV leading to significant differences at $1 \mathrm{GV}$. At low energies these differences can be compensated by other propagation phenomena like convection and/or reacceleration.

We perform additional fits for the BASE and the BASE + inj $+v_{A}-$ diff.brk setup in which we leave the half-height of the diffusion halo $z_{\mathrm{h}}$ as a free parameter varied between $2 \mathrm{kpc}$ and $10 \mathrm{kpc}$. From these fits we obtain the $\chi^{2}$ profile as function of $z_{\mathrm{h}}$ as shown in Fig. 4. We conclude that small halo heights below $\sim 3 \mathrm{kpc}$ are strongly disfavored when the LiBeBCNO data set is used, while the BCNO data set does not give constraints. This behavior is expected because of the radioactive isotope ${ }_{4}^{10} \mathrm{Be}$ which decays to $\mathrm{B}$ with a lifetime of about $1 \mathrm{My}$. Therefore, a comparison between $\mathrm{B}$ and Be can lift the degeneracy between $D_{0}$ and $z_{\mathrm{h}}$. The conclusions are slightly weakened when correlated AMS-02 data are considered. 


\section{Conclusion}

We perform a fit of AMS-02 CR data from Li to O. We have considered five different propagation frameworks. They differ with respect to the inclusion of breaks in the injection spectrum or in the diffusion coefficient at a few GV, and the presence of reacceleration. From a physical point of view, the two most different setups considered in this work are BASE and BASE+inj $+v_{A}-$ diff.brk. The first setup contains a break in the diffusion coefficient at a few GV and does not include reacceleration while the latter allows for reacceleration and has no break in the diffusion. We note that these two setups are also the ones with the smallest number of free parameters. It is a remarkable result that both setups are able to fit the precise AMS-02 data equally well. However, we stress that this conclusion critically depends on the inclusion of the sizable uncertainties in the fragmentation cross sections which are at the level of 20-30\%. To this aim we make use of Monte Carlo scanning techniques in order to handle the large $(>20)$ dimensionality of the parameter space. This allows us to vary and fit $\mathrm{CR}$ propagation parameters and nuisance parameters of the cross section at the same time. Thus, a crucial conclusion of this work is that further experimental efforts are mandatory to reduce cross section uncertainties so that to exploit in full the precision of CR data. Nonetheless, despite the sizable uncertainties we find some important robust conclusions. At intermediate energies the slope of the diffusion coefficient is constrained at $\delta \simeq 0.4-0.5$ pointing towards a Kraichnan model of turbulence. Furthermore, the combination of $\mathrm{Be}$ and $\mathrm{B}$ data strongly disfavors very small values below $3 \mathrm{kpc}$ for the half-height of the diffusion halo $z_{\mathrm{h}}$.

\section{Acknowledgments}

The work of M.K. is partially supported by the Swedish National Space Agency under contract 117/19 and the European Research Council under grant 742104, and by the Swedish Research Council under Contract No. 335-2014-7424. A.C. is supported by: "Departments of Excellence 2018-2022" grant awarded by the Italian Ministry of Education, University and Research (MIUR) L. 232/2016; Research grant "The Dark Universe: A Synergic Multimessenger Approach" No. 2017X7X85K, PRIN 2017, funded by MIUR; Research grant TAsP (Theoretical Astroparticle Physics) funded by INFN.

\section{References}

[1] AMS collaboration, The Alpha Magnetic Spectrometer (AMS) on the international space station: Part II - Results from the first seven years, Phys. Rept. 894 (2021) 1.

[2] M. Korsmeier and A. Cuoco, Galactic cosmic-ray propagation in the light of AMS-02: Analysis of protons, helium, and antiprotons, Phys. Rev. D94 (2016) 123019 [1607.06093].

[3] N. Tomassetti, Solar and nuclear physics uncertainties in cosmic-ray propagation, Phys. Rev. D96 (2017) 103005 [1707.06917].

[4] W. Liu, Y.-h. Yao and Y.-Q. Guo, Revisiting the Spatially Dependent Propagation Model with the Latest Observations of Cosmic-Ray Nuclei, Astrophys. J. 869 (2018) 176 [1802 . 03602].

[5] Y. Génolini et al., Cosmic-ray transport from AMS-02 boron to carbon ratio data: Benchmark models and interpretation, Phys. Rev. D99 (2019) 123028 [1904 . 08917]. 
[6] N. Weinrich, M. Boudaud, L. Derome, Y. Genolini, J. Lavalle, D. Maurin et al., Galactic halo size in the light of recent AMS-02 data, Astron. Astrophys. 639 (2020) A74 [2004.00441].

[7] C. Evoli, G. Morlino, P. Blasi and R. Aloisio, The AMS-02 beryllium and its implication for Cosmic Ray transport, 1910.04113.

[8] M. Boschini et al., Deciphering the local Interstellar spectra of secondary nuclei with GALPROP/HelMod framework and a hint for primary lithium in cosmic rays, 1911.03108.

[9] P.D.L.T. Luque, M.N. Mazziotta, F. Loparco, F. Gargano and D. Serini, Markov chain Monte Carlo analyses of the flux ratios of B, Be and Li with the DRAGON2 code, 2102 .13238.

[10] AMS collaboration, Observation of New Properties of Secondary Cosmic Rays Lithium, Beryllium, and Boron by the Alpha Magnetic Spectrometer on the International Space Station, Phys. Rev. Lett. 120 (2018) 021101.

[11] AMS collaboration, Observation of the Identical Rigidity Dependence of He, $C$, and $O$ Cosmic Rays at High Rigidities by the Alpha Magnetic Spectrometer on the International Space Station, Phys. Rev. Lett. 119 (2017) 251101.

[12] AMS collaboration, Precision Measurement of Cosmic-Ray Nitrogen and its Primary and Secondary Components with the Alpha Magnetic Spectrometer on the International Space Station, Phys. Rev. Lett. 121 (2018) 051103.

[13] M. Korsmeier and A. Cuoco, Implications of Lithium to Oxygen AMS-O2 spectra on our understanding of cosmic-ray diffusion, Phys. Rev. D 103 (2021) 103016 [2103.09824].

[14] A.W. Strong, I.V. Moskalenko and V.S. Ptuskin, Cosmic-ray propagation and interactions in the Galaxy, Ann. Rev. Nucl. Part. Sci. 57 (2007) 285 [astro-ph/0701517].

[15] D.A. Green, Constraints on the distribution of supernova remnants with Galactocentric radius, Mon. Not. Roy. Astron. Soc. 454 (2015) 1517 [1508. 02931].

[16] V.A. Dogiel, V.S. Berezinsky, S.V. Bulanov and V.S. Ptuskin, Astrophysics of cosmic rays, Elsevier Since Publischers B.V. (1990).

[17] E.S. Seo and V.S. Ptuskin, Stochastic reacceleration of cosmic rays in the interstellar medium, Astrophys. J. 431 (1994) 705.

[18] A.W. Strong, I.V. Moskalenko and O. Reimer, Diffuse continuum gamma-rays from the galaxy, Astrophys. J. 537 (2000) 763 [astro-ph/9811296].

[19] A.W. Strong, Recent extensions to GALPROP, 1507.05020.

[20] L.A. Fisk, Solar Modulation and a Galactic Origin for the Anomalous Component Observed in Low-Energy Cosmic Rays, Astrophys. J. 206 (1976) 333.

[21] Y. Genolini, D. Maurin, I.V. Moskalenko and M. Unger, Current status and desired precision of the isotopic production cross sections relevant to astrophysics of cosmic rays: $L i, B e, B, C$, and N, Phys. Rev. C98 (2018) 034611 [1803. 04686].

[22] F. Feroz, M.P. Hobson and M. Bridges, MultiNest: an efficient and robust Bayesian inference tool for cosmology and particle physics, Mon. Not. Roy. Astron. Soc. 398 (2009) 1601 [0809.3437].

[23] J. Heisig, M. Korsmeier and M.W. Winkler, Dark matter or correlated errors: Systematics of the AMS-02 antiproton excess, Phys. Rev. Res. 2 (2020) 043017 [2005 . 04237].

[24] L. Derome, D. Maurin, P. Salati, M. Boudaud, Y. Génolini and P. Kunzé, Fitting B/C cosmic-ray data in the AMS-02 era: A cookbook, Astron. Astrophys. 627 (2019) A158 [1904.08210]. 